\title{
Knockdown of IncRNA PVT1 inhibits prostate cancer progression in vitro and in vivo by the suppression of KIF23 through stimulating miR-15a-5p
}

Huijuan $\mathrm{Wu}^{1 *+} \mathbb{B}$, Xin $\operatorname{Tian}^{2 \dagger}$ and Chaoyang Zhu

\begin{abstract}
Background: Prostate cancer (PCa) greatly threatens men's lives, with high incidence and mortality. Recently, the research of long non-coding RNAs (IncRNAs) has made breakthroughs in the development of human cancers. This study aimed to figure out the role and action mechanism of IncRNA PVT1 (PVT1) in PCa.

Methods: The expression of PVT1, microRNA-15a-5p (miR-15a-5p) and kinesin family member 23 (KIF23) was detected by quantitative real-time polymerase chain reaction (qRT-PCR). Cell proliferation, apoptosis, migration and invasion were assessed by 3-(4,5-dimethyl-2-thiazolyl)-2,5-diphenyl-2-H-tetrazolium bromide (MTT), flow cytometry and transwell assays, respectively. The protein levels of KIF23 and proliferation, apoptosis, and epithelial-mesenchymal transition (EMT)-related markers were quantified by western blot. The relationship between miR-15a-5p and PVT1 or KIF23 was predicted by starBase v2.0 and verified by dual-luciferase reporter assay. Xenograft assay was conducted to determine the role of PVT1 in vivo.

Results: The expression of PVT1 and KIF23 was enhanced, while miR-15a-5p expression was reduced in PCa tissues and cells. PVT1 interference inhibited proliferation, migration and invasion but promoted apoptosis of PCa cells. MiR$15 a-5 p$ was a target of PVT1, and KIF23 was a target of miR-15a-5p. The inhibition of miR-15a-5p reversed the effects of PVT1 interference and suppressed the roles of KIF23 knockdown. KIF23 expression was regulated by PVT1 through miR-15a-5p. PVT1 interference blocked PCa progression in vivo.
\end{abstract}

Conclusion: PVT1 knockdown had effects on the progression of PCa by inhibiting the expression of KIF23 via enriching miR-15a-5p in vitro and in vivo, suggesting that PVT1 might be a novel biomarker for the treatment of PCa.

Keywords: PVT1, miR-15a-5p, KIF23, Prostate cancer

*Correspondence: 46408297@qq.com

'Huijuan Wu and Xin Tian contribute to this work equally as co-first authors

${ }^{1}$ Department of Telemedicine and Internet Medical Center, The Huaihe Hospital of Henan University, No. 115 Ximen Avenue, Kaifeng 475000, Henan, China

Full list of author information is available at the end of the article

\section{Highlights}

1. PVT1 and KIF23 are up-regulated, while miR-15a-5p is down-regulated in $\mathrm{PCa}$ tissues and cells.

2. PVT1 interference attenuates malignant activities of PCa cells in vitro.

3. PVT1 directly targets miR-15a-5p, and miR-15a-5p directly binds to KIF23.

4. PVT1 interference regulates PCa progression by the regulation of KIF23 and miR-15a-5p.

(c) The Author(s) 2020. This article is licensed under a Creative Commons Attribution 4.0 International License, which permits use, sharing, adaptation, distribution and reproduction in any medium or format, as long as you give appropriate credit to the original author(s) and the source, provide a link to the Creative Commons licence, and indicate if changes were made. The images or other third party material in this article are included in the article's Creative Commons licence, unless indicated otherwise in a credit line to the material. If material is not included in the article's Creative Commons licence and your intended use is not permitted by statutory regulation or exceeds the permitted use, you will need to obtain permission directly from the copyright holder. To view a copy of this licence, visit http://creativeco mmons.org/licenses/by/4.0/. The Creative Commons Public Domain Dedication waiver (http://creativecommons.org/publicdomain/ zero/1.0/) applies to the data made available in this article, unless otherwise stated in a credit line to the data. 
5. PVT1 knockdown impedes PCa development in vivo.

\section{Background}

Prostate cancer ( $\mathrm{PCa})$, a common malignancy in the male population, is an important cause of male mortality [1]. The occurrence of $\mathrm{PCa}$ is more prevalent in developed countries [2,3]. The complex mechanism of $\mathrm{PCa}$ initiation and development involves various factors, such as age, lifestyle, environment, and heredity $[1,4]$. Androgen deprivation therapy is the mainstream method for the treatment of $\mathrm{PCa}$ and has a considerable curative effect in the early stage $[5,6]$. In general, inappropriate screening schedules, low cure rates, and drug resistance are substantial burdens in the treatment of PCa [1]. Therefore, the underlying mechanisms associated with the development and progression of $\mathrm{PCa}$ and novel targeted specific biomarkers still require further investigation.

Long non-coding RNAs (lncRNAs), over 200 nucleotides in length, are a kind of RNA molecules with extensive functionality [7]. Although our current understanding of the role of lncRNAs is limited, existing reports reveal partial functions of lncRNAs involved in nuclear structural integrity, modulation of gene expression, chromatin remodeling, transcription and posttranscriptional processing [8]. Recently, the involvement of IncRNAs in cancer progression has attracted much attention [9]. In PCa, dozens of lncRNAs have been identified as biologically significant. For example, lncRNA SNHG20, with a high level in PCa tissues and cells, contributed to the proliferation and invasion of $\mathrm{PCa}$ cells [10]. LncRNA MALAT1 was also overexpressed in PCa, and its high expression was associated with tumor stage, drug resistance, tumorigenicity and progression of $\mathrm{PCa}$ [11]. LncRNA Plasmacytoma Variant Translocation 1 (PVT1), was well known to play a part in tumorigenesis in multiple cancer types [12-14]. In this study, a novel mechanism of PVT1 involving in the development of PCa was identified.

MicroRNAs (miRNAs), 18-24 nucleotides in length, generally play biological functions by acting as the downstream target of lncRNAs [15, 16]. As a kind of noncoding RNAs, the functional roles of miRNAs have been well investigated. In cancer, miRNAs serve as tumor suppressors or promoters by competitively modulating the expression of downstream specific target genes [16, 17]. Existing reports stated that miR-15a-5p participated in numerous cancers, such as breast cancer [18], papillary thyroid cancer [19], and endometrial cancer [20]. However, the function of miR-15a-5p in PCa was not fully elucidated, and related mechanism of miR-15a-5p in PCa was lacking. Kinesin family member 23 (KIF23) locates at the interzone of mitotic spindles and functions by driving microtubule movement [21]. Previous studies demonstrated that KIF23 had been identified as a novel therapeutic target in the treatment of lung cancer, pleural mesothelioma, and gastric cancer [22-24]. Unfortunately, the function of KIF23 in the progression of PCa was limited to mention yet. Accordingly, our study devoted to exploring the potential roles of miR-15a-5p and KIF23 in the tumorigenesis and progression of $\mathrm{PCa}$.

Here, we investigated the expression of PVT1 in PCa tissues and cell lines, as wells as the expression of miR15a-5p and KIF23. The specific function of PVT1 was determined in vitro and in vivo. The relationship between miR-15a-5p and PVT1 or KIF23 was confirmed, so as to provide a new sight and a novel mechanism for the progression of PCa.

\section{Materials and methods}

\section{Specimens}

This research acquired the approval of the Ethics Committee of the Huaihe Hospital of Henan University. A total of 25 paired PCa tissues and adjacent normal tissues were collected from the Huaihe Hospital of Henan University. After removal, all specimens were quickly placed into liquid nitrogen and stored at $-80{ }^{\circ} \mathrm{C}$ soon after. Each subject had signed the informed consent prior to the surgery. The clinicopathological characteristics of PCa patients were recorded in Additional file 1: Table S1.

\section{Cell lines and cell culture}

PCa cell lines (22RV1 and DU145), normal prostate epithelial cell line (RWPE-1) and embryonic kidney cells (293T) were all purchased from BeNa Culture Collection (Suzhou, China). According to the instruction, 22RV1 cells were maintained in 90\% Roswell Park Memorial Institute 1640 (RPMI 1640; Sigma, St. Louis, MO, USA) containing 10\% fetal bovine serum (FBS; Sigma). DU145, RWPE-1 and 293T cells were cultured in 90\% Dulbecco's Modified Eagle Medium (DMEM; Sigma) containing 10\% FBS (Sigma). These cell lines were maintained at $37{ }^{\circ} \mathrm{C}$ conditions containing $5 \% \mathrm{CO}_{2}$.

\section{Quantitative real-time polymerase chain reaction (qRT-PCR)}

MiRNeasy kit (Qiagen, Hilden, Germany) was used for RNA extraction. For PVT1 and KIF23, OneStep RT-PCR Kit (Qiagen) was adapted for reverse transcription (RT) reactions to assemble complementary DNA (cDNA). For miR-15a-5p, miScript II RT Kit (Qiagen) was used for synthesizing cDNA. QuantiFast SYBR Green PCR Kit (Qiagen) was used to perform qRT-PCR using the Step One Plus real-time PCR system (Applied Biosystems, Foster City, CA, USA). Relative expression was normalized by $\beta$-actin or small nuclear RNA U6 and calculated 
using the $2^{-\Delta \Delta C t}$ method. The primers were displayed as below: PVT1: 5' - CAGCACTCTGGACGGAC- $3^{\prime}$ (forward) and 5'- CAACAGGAGAAGCAAACA-3' (reverse); KIF23: 5'-AGACAGAAGGCGAGGGATG-3' (forward) and $5^{\prime}$ - GGAGACGAATTGGTGGTGC-3' (reverse); miR-15a-5p: 5'- TAGCAGCACATAATGGTT TGT-3' (forward) and 5' - GCGAGCACAGAATTAATA CGAC- $3^{\prime}$ (reverse); $\beta$-actin: $5^{\prime}$-TGGCACCCAGCACAA TGAA-3' (forward) and $5^{\prime}$-CTAAGTCATAGTCCGCCT AGAAGCA-3' (reverse); U6: 5' - CAGCACATATACTAA AATTGGAACG- $3^{\prime}$ (forward) and $5^{\prime}$ - ACGAATTTG CGTGTCATCC-3' (reverse).

\section{Cell transfection}

For PVT1 downregulation, small interference RNA (siRNA) against PVT1 (si-PVT1) and its negative control (si-NC) were assembled by Sangon Biotech (Shanghai, China). For stable PVT1 knockdown, lentiviral vector (lenti-short hairpin sh-PVT1) and its negative control (sh-NC) were obtained from Genechem (Shanghai, China). For miR-15a-5p inhibition or enrichment, miR-15a-5p inhibitor (5'-CACAAACCAUUAUGUGCU GCUA-3') or miR-15a-5p mimic (sense: 5'-UAGCAG CACAUAAUGGUUUGUG- $3^{\prime}$ and antisense: $5^{\prime}$-CAA ACCAUUAUGUGCUGCUAUU-3') and negative control (inhibitor NC (5'-CAGUACUUUUGUGUAGUACAA$3^{\prime}$ ) or miR-NC (sense: 5'-UUCUCCGAACGUGUCACG UTT- ${ }^{\prime}$ and antisense: $5^{\prime}$-ACGUGACACGUUCGGAGA ATT-3')) were purchased from Ribobio (Guangzhou, China). For KIF23 knockdown, siRNA against KIF23 (si-KIF23) and si-NC were also constructed by Sangon Biotech. The 22RV1 and DU145 cells were subjected to transfection with above items using Lipofectamine 2000 (Invitrogen, Carlsbad, CA, USA). Following experiments were conducted at $48 \mathrm{~h}$ post-transfection.

\section{3-(4,5-Dimethyl-2-thiazolyl)-2,5-diphenyl-2-H-tetrazolium bromide (MTT) assay}

22RV1 and DU145 cells with different transfection were seeded into 96-well plates (Corning Costar, Corning, NY, USA) at a density of $5 \times 10^{3}$ cells per well. Then $10 \mu \mathrm{L}$ MTT solution (Beyotime, Shanghai, China) was pipetted into each well at $0 \mathrm{~h}, 24 \mathrm{~h}, 48 \mathrm{~h}$ and $72 \mathrm{~h}$ for another $4 \mathrm{~h}$ at $37^{\circ} \mathrm{C}$. After that, the dimethyl sulfoxide (DMSO; Beyotime) was added into each well to dissolve the formazan. The absorbance was measured at $490 \mathrm{~nm}$ using the Multiskan Ascent (Thermo Fisher Scientific, Waltham, MA, USA) to assess cell proliferation.

\section{Flow cytometry assay}

22RV1 and DU145 cells with different transfection were seeded into 6 -well plates. Then, $0.25 \%$ trypsin was used to deal with the cells. Next, the cells after washing with phosphate buffer saline (PBS) were detected by Annexin V-FITC Apoptosis Detection Kit (KeyGEN, Nanjing, China). In brief, cells were resuspended by $500 \mu \mathrm{L}$ binding buffer and then incubated by $5 \mu \mathrm{L}$ Annexin V-fluorescein isothiocyanate (FITC) and propidium iodide (PI) for 15 min without light. Finally, the apoptotic cells were analyzed using flow cytometer (BD Biosciences, San Jose, CA, USA).

\section{Transwell assay}

The abilities of migration and invasion were assessed using 6-well transwell chambers (Corning Costar). Briefly, cells $\left(1 \times 10^{5}\right.$ cells $\left./ \mathrm{mL}\right)$ were resuspended in fresh DMEM or RPMI1640 containing 10\% FBS. Then, the suspensions were pipetted into the upper chambers, and DMEM or RPMI1640 containing 10\% FBS was added into the lower chambers. After culturing for $24 \mathrm{~h}$, the migrated cells in the low surface were fixed with formaldehyde and stained with $5 \%$ crystal violet. The cells were counted in five random fields using a microscope (Olympus, Tokyo, Japan) with magnification of $100 \times$. Noteworthily, the ability of cell invasion was also assessed using the same method, except that the upper transwell chambers needed to be coated with Matrigel (Corning Costar) before the inoculation.

\section{Western blot}

Western blot analysis was executed based on the methods described previously [25]. The primary antibodies against Vimentin (ab193555; 1:1000; Abcam, Cambridge, MA. USA), E-Cadherin (E-cad) (ab133597; 1:1000; Abcam), Caspase-3 (ab13847; 1:1000; Abcam), CyclinD1 (ab16663; 1:1000; Abcam), KIF23 (PA5-31773; 1:1000; Invitrogen) and $\beta$-actin (ab8227; 1:5000; Abcam) were used in this study, and goat anti-rabbit antobodies (ab205718; 1:5000; Abcam) were acted as the second antibodies. The protein blots were quantified using the Image J software (version 1.46; National Institutes of Health, Bethesda, MA, USA).

\section{Bioinformatics analysis and dual-luciferase reporter assay}

The online tool starBase v2.0 (http://starbase.sysu.edu. $\mathrm{cn} /$ ) was used to predict the potential target genes and analyze the specific binding sites.

The relationship between miR-15a-5p and PVT1 or KIF23 was verified by dual-reporter assay. In brief, the fusion plasmids (pRL-CMV, Promega, Madison, WI, USA) containing the sequences of PVT1 wild type (WTPVT1) with the miR-15a-5p binding sites or PVT1 mutant (MUT-PVT1) with the mutated miR-15a-5p binding sites, or containing the sequences of wild type KIF23 $3^{\prime}$ untranslated region (UTR) (KIF23 3' UTRWT) with the miR-15a-5p binding sites or mutant KIF23 3' UTR (KIF23 3' UTR-MUT) with the mutated 
miR-15a-5p binding sites were obtained from Sangon Biotech. The 293T cells were cotransfected with above fusion plasmids and miR-15a-5p or miR-NC, respectively. After $48 \mathrm{~h}$-transfection, the luciferase activity was determined using the Dual-Luciferase Reporter Assay Kit (Promega).

\section{Xenograft assay}

All animal procedures were approved by the Animal Care and Use Committee of the Huaihe Hospital of Henan University. A total of $12 \mathrm{BALB} / \mathrm{c}$ nude mice (6-week-old, male) were purchased from HFK bioscience Co., LTD (Beijing, China). DU145 cells packaged with lentiviral vector containing sh-PVT1 or sh-NC were planted into 24-well plates $\left(5 \times 10^{4}\right.$ cells/well $)$. Then, different concentrations of puromycin $(1,2,5,10$ and $15 \mu \mathrm{g} / \mathrm{mL})$ were added into different wells, and the cell viability was monitored every day to screen stable cells with transfection. DU145 cells with stable sh-PVT1 or sh-NC transfection were subcutaneously injected into the right flank of mice ( $n=6$ per group). Ten days after inoculation, the tumor volume was measured every 4 days based on the formula: length $\times$ width $^{2} \times 0.5$. Afterwards, the mice were killed after 34 days, and the tumor tissues were collected for the following analyses.

\section{Statistical analysis}

The data were analyzed by GraphPad Prism 5.01 (GraphPad Software, Inc., La Jolla, CA, USA) and presented as the mean \pm standard deviation (SD). Each experiment was repeated at least 3 times. The differences were distinguished by Student's $t$-test or one-way analysis of variance (ANOVA). The correlation analysis was performed according to the Spearman's correlation coefficient.
$P<0.05$ was considered to be a statistically significant difference.

\section{Result}

PVT1 was up-regulated in PCa tissues and cells

To define the expression of PVT1 in PCa, qRT-PCR was carried out. The result showed that PVT1 was highly expressed in PCa tissues $(n=25)$ compared with that in normal tissues $(n=25)$ (Fig. 1a). Likewise, the expression of PVT1 in PCa cell lines (22RV1 and DU145) was higher than that in a normal prostate epithelial cell line (RWPE1) (Fig. 1b). The data indicated that PVT1 was aberrantly regulated in $\mathrm{PCa}$ and might play a part in the development of PCa.

\section{Interference of PVT1 inhibited proliferation, migration and invasion but induced apoptosis of PCa cells}

To determine the role of PVT1 on cell proliferation, apoptosis, migration and invasion, the endogenous level of PVT1 was knocked down in 22RV1 and DU145 cells. The efficiency of PVT1 interference was checked using qRT-PCR, and the data displayed that PVT1 was noticeably down-regulated in PCa cells transfected with si-PVT1 (Fig. 2a). The proliferation of 22RV1 and DU145 cells was significantly repressed in response to PVT1 interference by MTT assay (Fig. 2b). Flow cytometry assay presented that interference of PVT1 notably increased the apoptosis rate of 22RV1 and DU145 cells compared with sh-NC (Fig. 2c). In addition, transwell assay was conducted to determine the effects on cell invasion and migration. The data presented that interference of PVT1 notably reduced the number of migrated and invaded cells (Fig. 2d). Additionally, several protein markers of proliferation, apoptosis, migration and invasion were quantified, and the result suggested that the levels of Vimentin
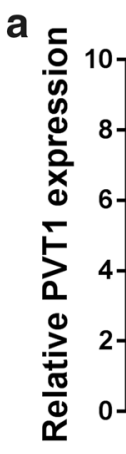

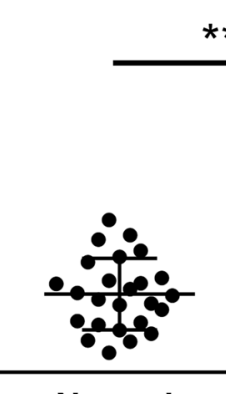

Normal

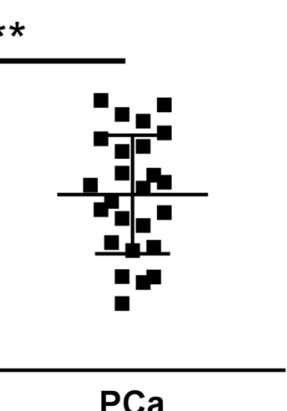

\section{b}

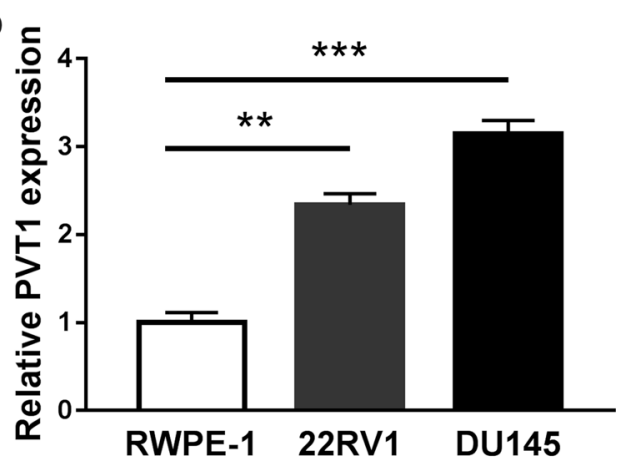

Fig. 1 The expression of PVT1 was enhanced in PCa tissues and cell lines. a The expression of PVT1 in PCa tissues $(n=25)$ and adjacent normal tissues $(n=25)$ was detected by qRT-PCR. $\mathbf{b}$ The expression of PVT1 in 22RV1, DU145 and RWPE-1 cells was also detected by qRT-PCR. ${ }^{* *} P<0.01$, ***P $<0.001$ 


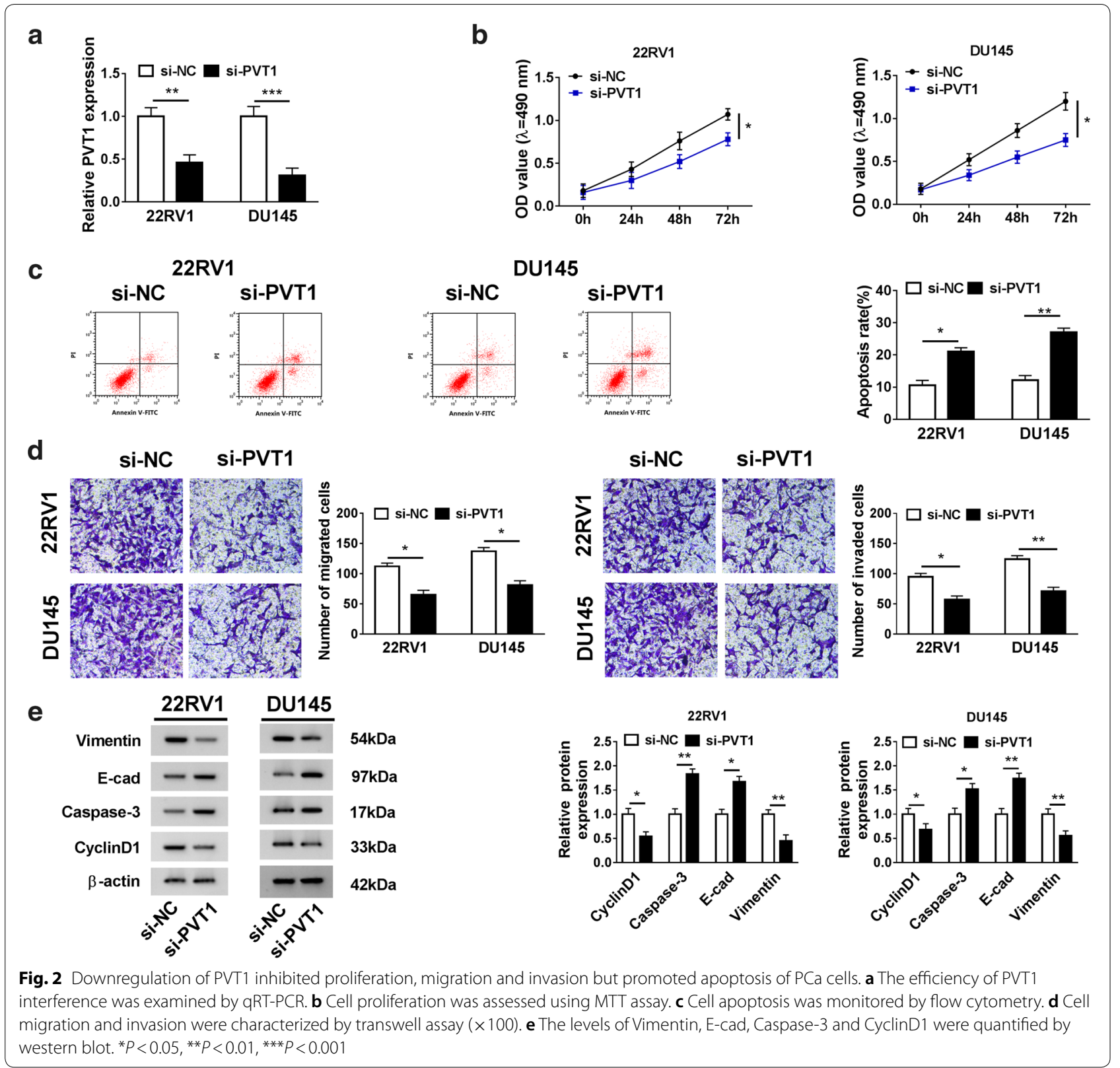

and CyclinD1 were prominently decreased, while the levels of Caspase-3 and E-cad were pronouncedly increased in 22RV1 and DU145 cells transfected with si-PVT1 (Fig. 2e). Abovementioned analyses suggested that PVT1 knockdown suppressed the malignant phenotypes of $\mathrm{PCa}$ cells.

\section{MiR-15a-5 $p$ was targeted by PVT1, and its inhibition} reversed the effects of PVT1 interference in PCa cells To explore the potential mechanism of PVT1 in the development of $\mathrm{PCa}$, the potential target miRNAs of PVT1 were predicted by starBase v2.0, including
miR-515-5p, miR-24-3p, miR-512-3p, miR-15a-5p, miR21-5p and miR-17-5p. Compared with other miRNAs, the expression of miR-15a-5p decreased more in PCa tissues, and miR-15a-5p expression increased more in $\mathrm{PCa}$ cells transfected with si-PVT1 (Additional file 2: Figure S1). Herein, miR-15a-5p was selected for further analyses. The expression of miR-15a-5p was strongly declined in PCa tissues and cell lines relative to normal tissues and cell line, respectively (Fig. 3a, b). In addition, we found that the expression of miR-15a-5p was negatively correlated to PVT1 expression in PCa tissues (Fig. 3c). According to the predicted binding site between miR-15a-5p 
(See figure on next page.)

Fig. 3 MiR-15a-5p was a target of PVT1, and miR-15a-5p inhibition reversed the effects of PVT1 interference in PCa cells. a, b The expression of miR-15a-5p was measured in PCa tissues and cell lines by qRT-PCR. c The correlation between miR-15a-5p expression and PVT1 expression was analyzed according to Spearman's correlation coefficient. $\mathbf{d}$ The binding sites between PVT1 and miR-15a-5p were predicted by starBase V2.0, and their relationship was verified by dual-luciferase reporter assay. e The expression of miR-15a-5p was affected by the change of PVT1 expression. $\mathbf{f}$ The expression of miR-15a-5p, $\mathbf{g}$ cell proliferation, $\mathbf{h}$ apoptosis, $\mathbf{i}$ migration $(\times 100), \mathbf{j}$ invasion $(\times 100)$ and $\mathbf{k}$ the levels of Vimentin, E-cad, Caspase-3 and CyclinD1 were determined in PCa cells transfected with si-PVT1, si-NC, si-PVT1 + miR-15a-5p inhibitor and si-PVT1 + inhibitor NC, respectively. ${ }^{*} P<0.05,{ }^{* *} P<0.01,{ }^{* * *} P<0.001$

and PVT1, the mutant sequence fragment of PVT1 harboring mutated binding site was designed (Fig. 3d). Meanwhile, dual-luciferase reporter assay manifested that the luciferase activity was obviously diminished in 293T cells transfected with miR-15a-5p and WT-PVT1 compared with that in 293T cells transfected with WTPVT1 and miR-NC, while the luciferase activity did not change with the transfection of miR-15a-5p and MUTPVT1 (Fig. 3d). In order to verify the influence of PVT1 on miR-15a-5p expression, the expression of miR-15a-5p in 22RV1 and DU145 cells transfected with si-PVT1 or si-NC was observed by qRT-PCR. As shown in Fig. 3e, cells with si-PVT1 transfection showed significantly increased the expression of miR-15a-5p. Afterwards, the miR-15a-5p inhibitor was used to perform rescue experiments. The result of qRT-PCR appeared that the increased expression of miR-15a-5p induced by si-PVT1 was strikingly debilitated in cells with the cotransfection of miR-15a-5p inhibitor (Fig. 3f). MTT assay showed that si-PVT1-inhibited proliferation was recovered by miR15a-5p inhibition (Fig. 3g). Flow cytometry assay pointed out that si-PVT1-induced apoptosis was suppressed by miR-15a-5p inhibition (Fig. 3h). Transwell assay maintained that the number of migrated and invaded cells was blocked by si-PVT1 but restored by si-PVT1 + miR15a-5p inhibitor (Fig. 3i, j). Additionally, western blot showed that the levels of Vimentin and CyclinD1 inhibited by si-PVT1 were regained by si-PVT1 + miR-15a-5p inhibitor, while the levels of E-cad and Caspase-3 promoted by si-PVT1 were restrained by si-PVT1+ miR15a-5p inhibitor (Fig. 3k). These findings hinted that miR-15a-5p was a direct target of PVT1, and miR-15a-5p inhibition could recover the malignant phenotypes of PCa cells suppressed by PVT1 interference.

\section{KIF23 was a target of miR-15a-5p, and miR-15a-5p regulated its expression}

To further explore the action mechanism of PVT1 in the development of $\mathrm{PCa}$, the underlying target mRNAs of miR-15a-5p were screened and identified. Firstly, we monitored that the expression of KIF23 was significantly enhanced in $\mathrm{PCa}$ tissues compared with that in normal tissues at both mRNA and protein levels (Fig. 4a, b). As well, the expression of KIF23 was notably elevated in PCa cell lines compared with that in normal cell line at both mRNA and protein levels (Fig. 4c, d). Spearman's correlation coefficient indicated that miR-15a-5p expression was negatively correlated with KIF23 expression (Fig. 4e). Bioinformatics tool starBase v2.0 concluded that a special binding site existed between miR-15a-5p and KIF23 3' UTR (Fig. 4f). Dual-luciferase reporter assay presented that miR-15a-5p mimic transfection substantially reduced the luciferase activity in $293 \mathrm{~T}$ cells transfected with KIF23 3' UTR-WT compared with that in 293T cells transfected with KIF23 $3^{\prime}$ UTR-MUT, and RNA pull-down assay presented that Bio-miR-15a-5p largely enriched the abundance of KIF23 in the beads (Fig. 4g). Furthermore, we observed that the expression of KIF23 plummeted with the enrichment of miR-15a-5p at both mRNA and protein levels (Fig. $4 \mathrm{~h}, \mathrm{i}$ ). These data summarized that miR-15a-5p interacted with KIF23 and modulated the expression of KIF23.

\section{The impacts of KIF23 knockdown in PCa cells were inhibited by miR-15a-5p inhibitor}

To address the effects of the interaction between miR-15a-5p and KIF23, 22RV1 and DU145 cells were introduced with si-KIF23 and si-KIF23+miR-15a-5p inhibitor, respectively, si-NC and si-KIF23+inhibitor $\mathrm{NC}$ as the control. Firstly, the expression of KIF23 was detected, and the result showed that KIF23 was remarkably down-regulated in cells transfected with si-KIF23 but thereupon up-regulated with the transfection of siKIF23 + miR-15a-5p inhibitor at both mRNA and protein levels (Fig. 5a, b). Then, the ability of cell proliferation was inhibited by si-KIF23 but recovered by si-KIF23 + miR15a-5p inhibitor (Fig. 5c). The apoptosis rate of 22RV1 and DU145 cells was stimulated by KIF23 knockdown but blocked by miR-15a-5p inhibition (Fig. $5 \mathrm{~d}$ ). The number of migrated and invaded cells was decreased by the transfection of si-KIF23 but reincreased by the transfection of si-KIF23 + miR-15a-5p inhibitor (Fig. 5e, f). Additionally, the levels of Vimentin and CyclinD1 were reduced in cells transfected with si-KIF23 but enriched in cells transfected with si-KIF23 + miR-15a-5p inhibitor, while the levels of E-cad and Caspase-3 were on the contrary (Fig. 5g). Above data implied that miR-15a-5p inhibition could abolish the role of KIF23 knockdown. 


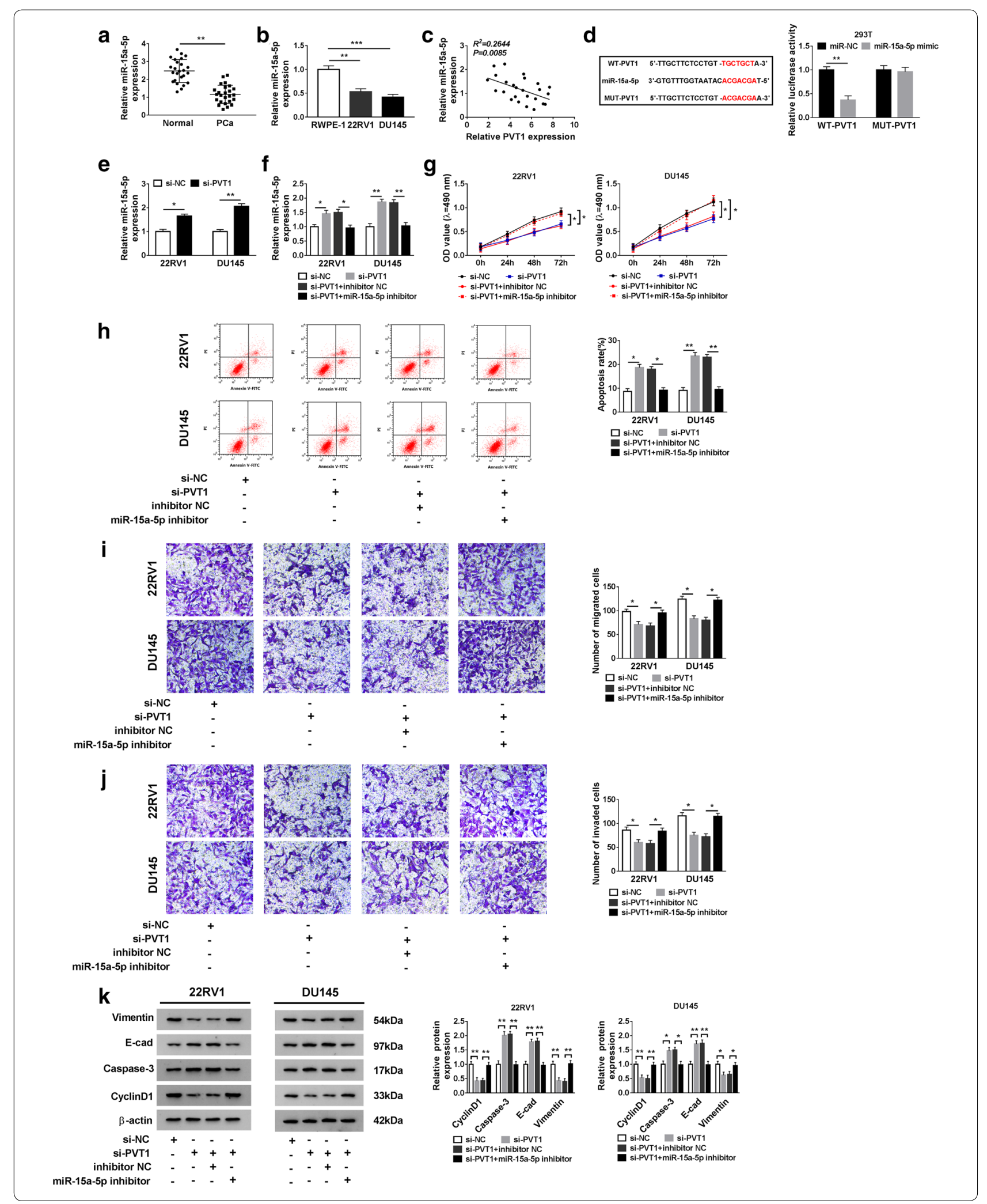




\section{(See figure on next page.)}

Fig. 4 KIF23 was up-regulated in PCa tissues and cell lines and targeted by miR-15a-5p. a, b The expression of KIF23 at mRNA and protein levels in PCa tissues and normal tissues was measured by qRT-PCR and western blot, respectively. $\mathbf{c}$, $\mathbf{d}$ The expression of KIF23 at mRNA and protein levels in PCa cells and normal cells was measured by qRT-PCR and western blot, respectively. e The expression of miR-15a-5p was negatively correlated with KIF23 expression. f The binding sites between miR-15a-5p and KIF23 $3^{\prime}$ UTR were forecasted by starBase v2.0. $\mathbf{g}$ The interaction between miR-15a-5p and KIF23 was confirmed by dual-luciferase reporter assay. $\mathbf{h}$, $\mathbf{i}$ The expression of KIF 23 was modulated by miR-15a-5p expression at both mRNA and protein levels. ${ }^{*} P<0.05,{ }^{* *} P<0.01,{ }^{* *} P<0.001$

\section{PVT1 regulated the expression of KIF23 through miR-15a-5p}

To further investigate whether PVT1 regulated KIF23 through miR-15a-5p, 22RV1 and DU145 cells were inserted with si-PVT1 and si-PVT1 + miR-15a-5p inhibitor, respectively, si-NC and si-PVT1+inhibitor NC as the control. The consequence of qRT-PCR and western blot displayed that the expression of KIF23 depleted by si-PVT1 was restored by si-PVT1 + miR-15a-5p inhibitor compared to corresponding control at both mRNA and protein levels (Fig. 6a, b). Furthermore, rescue experiments for KIF23 overexpression rescuing the effects of PVT1 knockdown were shown in Additional file 3: Figure S2. The data confirmed that PVT1 exerted its role in PCa progression by inducing KIF23 through mediating miR-15a-5p.

\section{PVT1 knockdown impeded tumor progression in vivo}

To examine the role of PVT1 on tumorigenesis in vivo, DU145 cells with stable PVT1 knockdown were inoculated into the subcutaneous tissues of nude mice. The efficiency of PVT1 knockdown in DU145 cells transfected with sh-PVT1 was displayed in Additional file 4: Figure S3, the data showed that the expression of PVT1 was remarkably declined in DU145 cells transfected with sh-PVT1 compared to sh-NC. The tumor volume, recorded every 4 days at 10 days post-injection, was obviously decreased in sh-PVT1-injected groups relative to sh-NC (Fig. 7a). The tumor weight was measured at the end, and the data indicated that the tumor weight was weaker in sh-PVT1-injected groups relative to sh-NC groups (Fig. 7b). Whereafter, the expression of PVT1, miR-15a-5p and KIF23 was detected in removed tumor tissues, and we found the expression of PVT1 and KIF23 was significantly declined, while miR-15a-5p expression was strengthened by qRT-PCR analysis (Fig. 7c). The expression of KIF23 at the protein level was consistent with its mRNA level (Fig. 7d). Moreover, western blot analysis manifested that the levels of Vimentin and CyclinD1 were markedly weakened in sh-PVT1 groups compared with that in sh-NC groups, while the levels of E-cad and Caspase-3 were reinforced in shPVT1 groups relative to that in sh-NC groups (Fig. 7e). These data ascertained that PVT1 knockdown blocked tumorigenesis and progression through the downregulation of KIF23 and the upregulation of miR-15a-5p in vivo.

\section{Discussion}

PCa accounts for 19\% of all new cancer cases and 9\% of all cancer-related deaths, according to the cancer statistics report [26]. The overall survival rate for PCa patients is unsatisfactory within 5 years. Hence, the identification of novel mechanisms and therapeutic biomarkers is needed for the improvement of PCa. In this study, we observed that PVT1 was aberrantly up-regulated in PCa, and PVT1 downregulation inhibited the malignant phenotypes of PCa cells in vitro and in vivo. The targeted relationship between miR-15a-5p and PVT1 or KIF23 was validated, and we found that PVT1 regulated the expression of KIF23 through miR-15a-5p, suggesting the regulatory effects of PVT1/miR-15a-5p/KIF23 axis in the progression of $\mathrm{PCa}$.

The research of lncRNAs in RNA biology is noticeable, and the exploration of certain lncRNAs in PCa leads us to determine the role of them as diagnostic, treatment and prognostic biomarkers [27]. Several previous studies mentioned the partial functions of PVT1 in PCa. For example, PVT1 knockdown blocked the proliferation and metastasis of PCa cells, partly via reducing the phosphorylated role of p38 [28]. PVT1 contributed to migration and invasion of $\mathrm{PCa}$ cells, which attributed to the activation of epithelial-mesenchymal transition (EMT) by the regulation of miR-186 and Twist1 [29]. PVT1 was up-regulated in PCa, and PVT1 regulated cell viability and apoptosis through the modulation of miR-146a [30]. These findings indicated that the action mechanisms of PVT1 in PCa were complex and diverse. Consistent with these studies, PVT1 was also highly expressed in PCa tissues and cells in our research. Besides, functional analyses deemed that interference of PVT1 ameliorated the malignant activities of $\mathrm{PCa}$ cells, suggesting the carcinogenic effect of PVT1.

To further understand the manner of PVT1 action, we attempted to seek for a novel mechanism of PVT1 in the progression of $\mathrm{PCa}$. Through the prediction of bioinformatics and the verification of dual-luciferase reporter assay, miR-15a-5p was identified as a target of PVT1. Previous studies concluded that miR-15a was downregulated in $\mathrm{PCa}$ tissues and cells, and its enrichment 


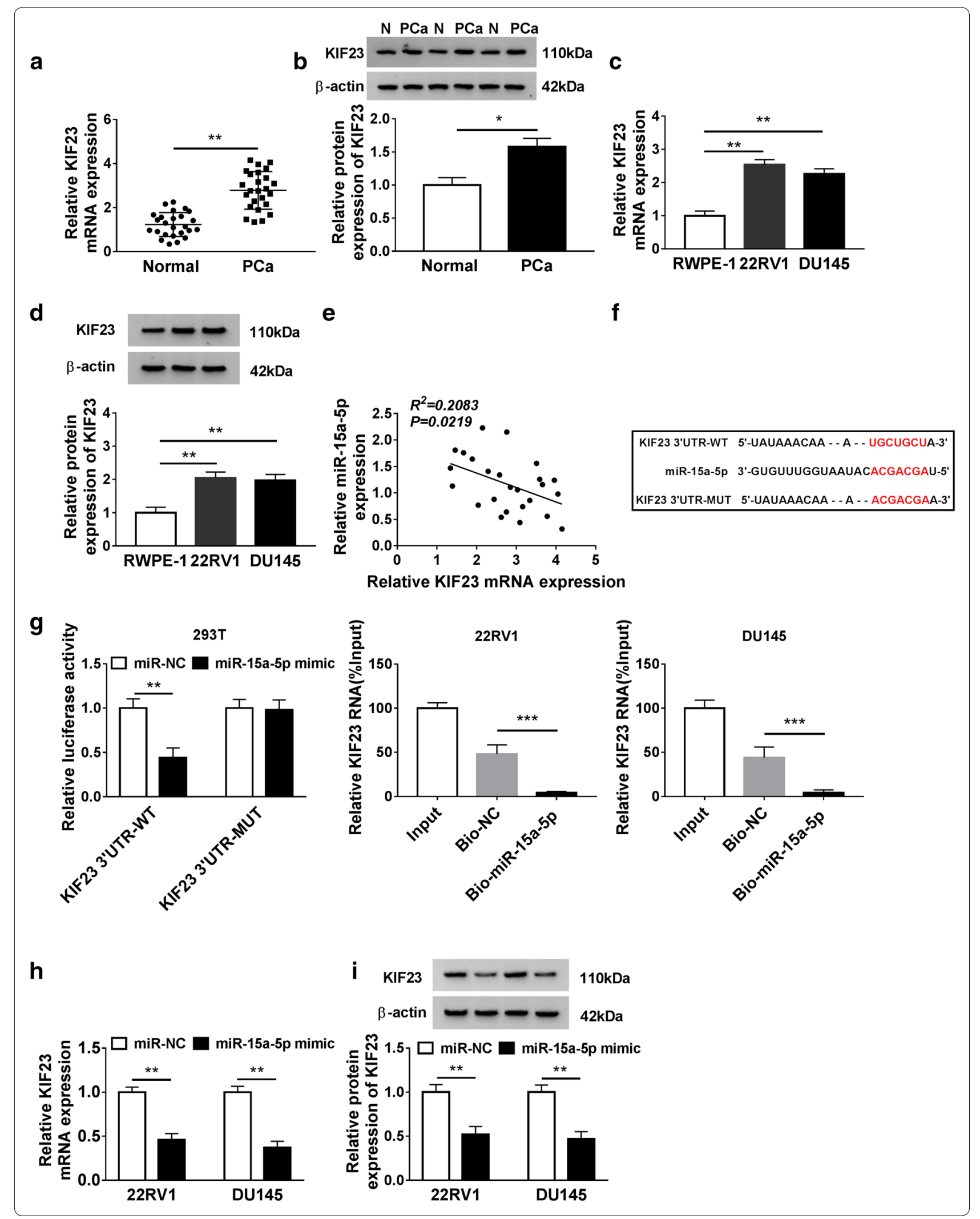




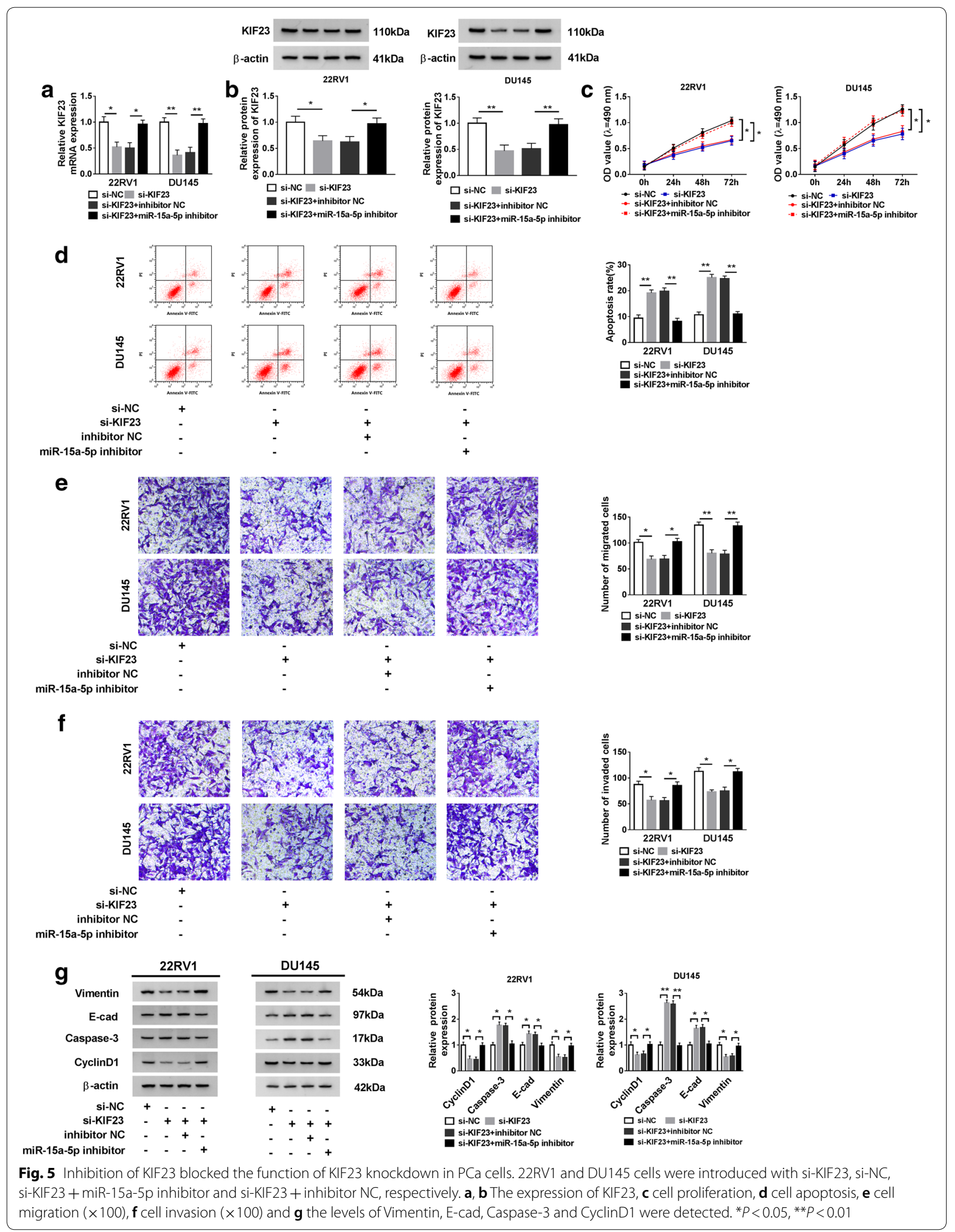




\section{a}

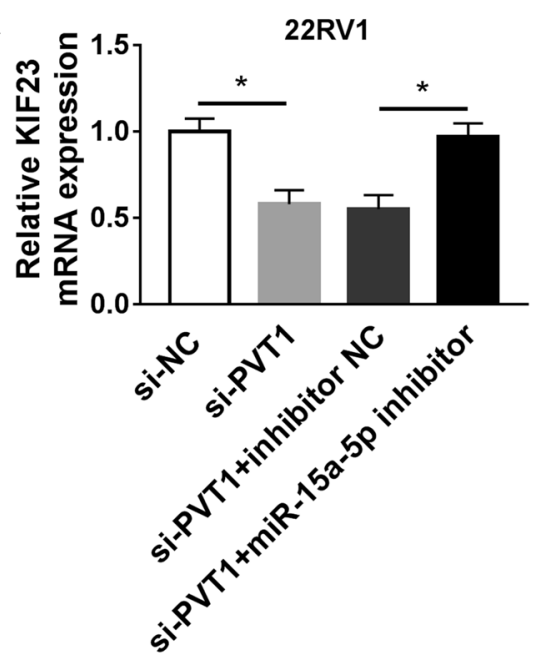

b

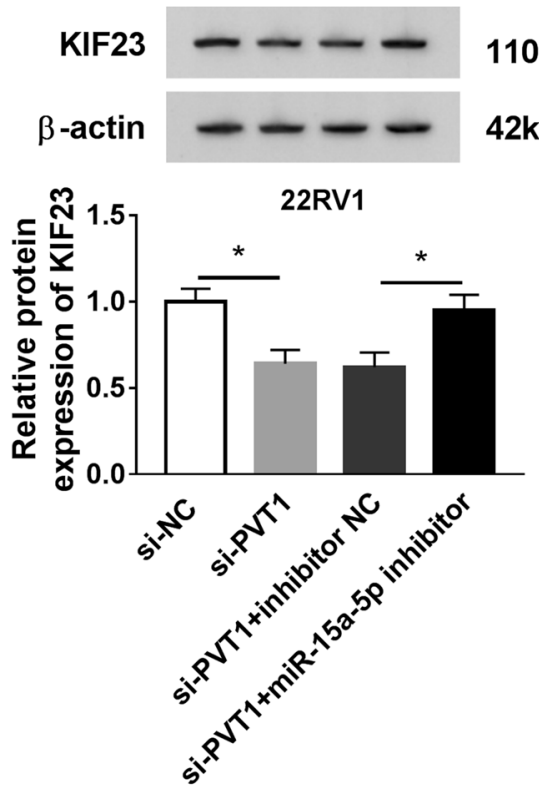

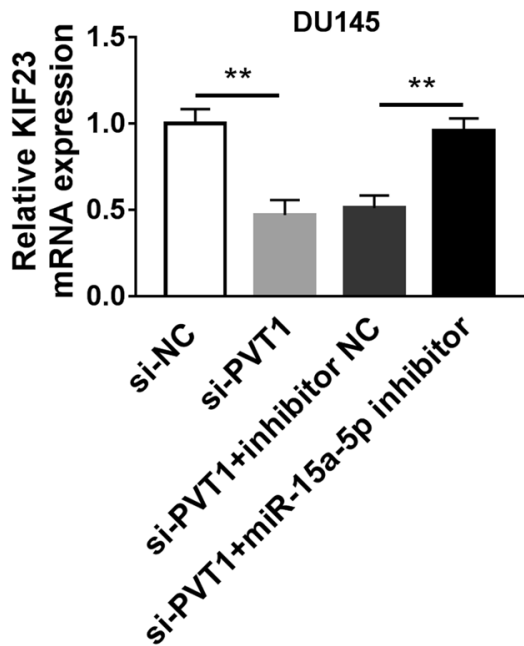
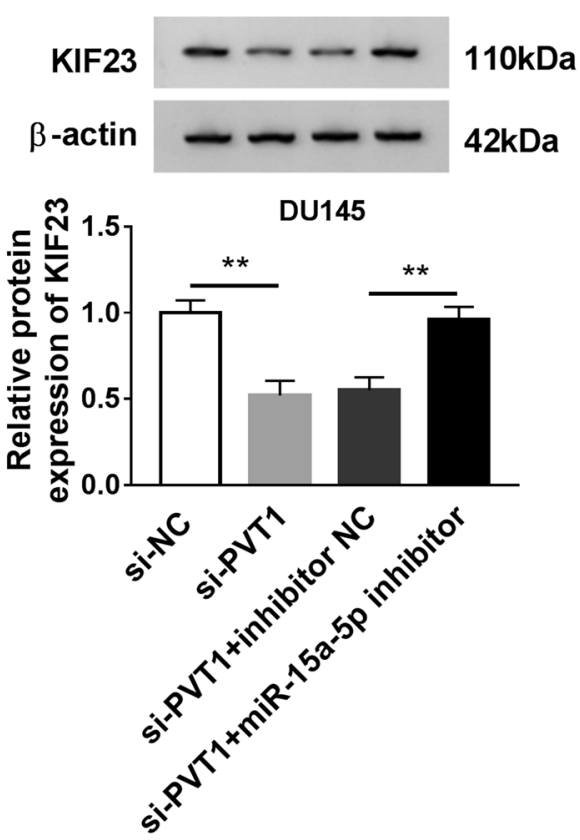

Fig. 6 The expression of KIF23 was regulated by PVT1 through miR-15a-5p in PCa cells. $\mathbf{a}$, $\mathbf{b}$ The expression of KIF23 at mRNA and protein levels was checked in 22RV1 and DU145 cells transfected with si-PVT1 and si-PVT1 + miR-15a-5p inhibitor, respectively, si-NC or si-PVT1 + inhibitor NC as the control. ${ }^{*} P<0.05,{ }^{* *} P<0.01$

could suppress invasion and proliferation of PCa cells $[31,32]$. In combination with the performance of miR$15 a-5 p$ in other cancers $[19,20,33]$, we deduced that miR-15a-5p might act as a tumor suppressor to inhibit the malignant behaviors of PCa. In agreement with these studies, we observed that the expression of miR-15a-5p was decreased in $\mathrm{PCa}$, and its inhibition could eliminate the effects of PVT1 interference in PCa cells. Interestingly, miR-15a-5p was proved as an oncogene in certain cancers, including cervical cancer [34] and colorectal adenocarcinoma [35]. This might be due to the different expression patterns of miR-15a-5p in diverse cancer types.

KIF23 was screened and confirmed as a direct target of miR-15a-5p. KIF23 was reported to be up-regulated in numerous cancers, leading to the accelerative cell proliferation, migration, poor prognostic and other harmful acts $[23,24,36]$. In PCa, the involvement of other members of the kinesin family in PCa had been expounded, such as KIF11, KIFC1, and KIF4A [37-39]. All of them were overexpressed in $\mathrm{PCa}$ and predicted poor prognosis. Consistent with the above findings, KIF23 was also highly 


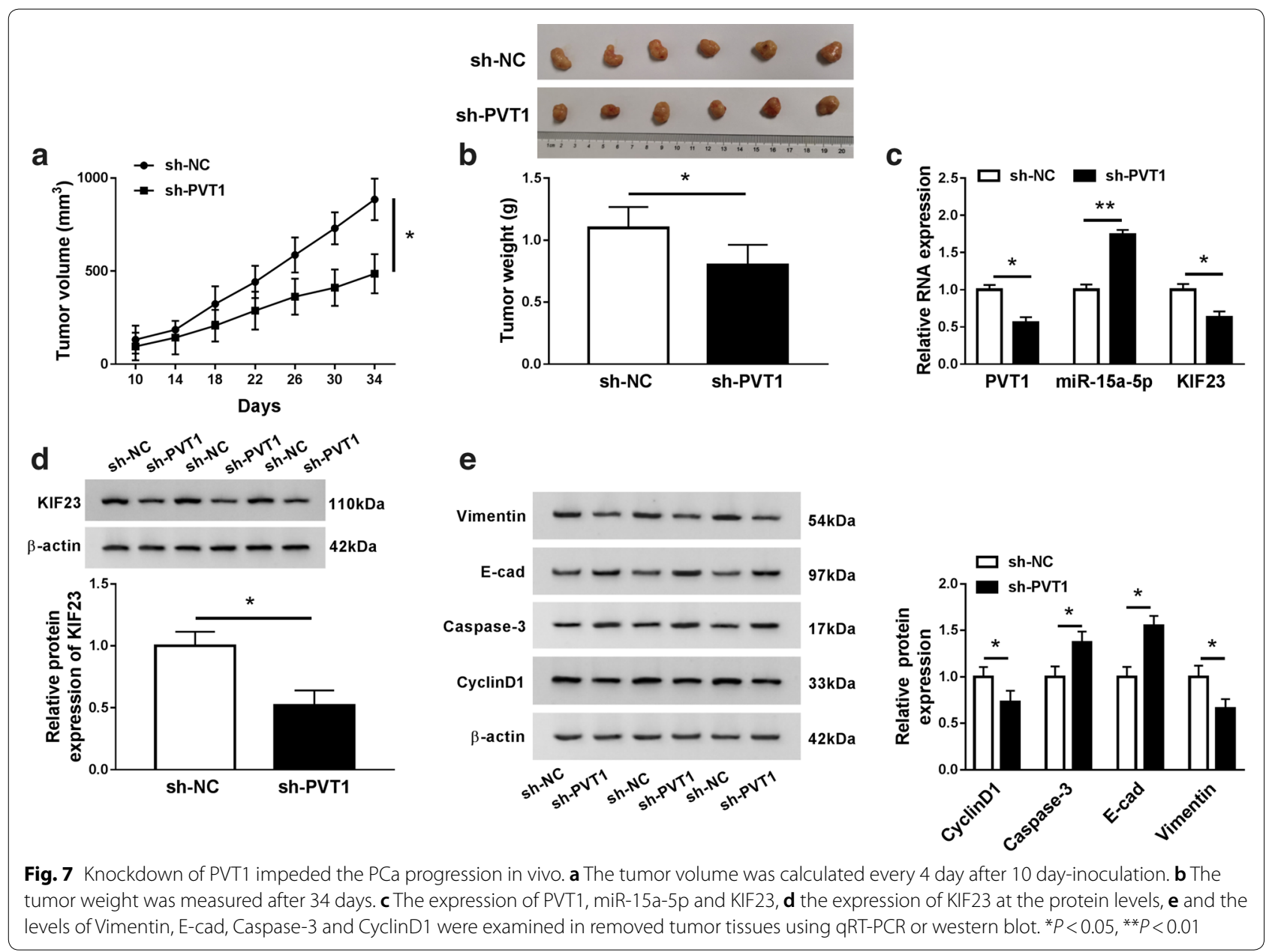

expressed in PCa tissues and cells through qRT-PCR and western blot analyses. Besides, KIF23 knockdown attenuated proliferation, migration and invasion but promoted apoptosis of PCa cells, implying that KIF23 was a tumor promoter in $\mathrm{PCa}$.

\section{Conclusion}

Collectively, the expression of PVT1 and KIF23 was reinforced in $\mathrm{PCa}$, while the expression of miR-15a-5p was declined. Knockdown of PVT1 suppressed the progression of PCa both in vitro and in vivo by mediating the miR-15a-5p/KIF23 network. Our present study provided a novel mechanism of $\mathrm{PCa}$ progression and broadened horizons to PVT1 as a biomarker for the treatment of PCa.

\section{Supplementary information}

Supplementary information accompanies this paper at https://doi. org/10.1186/s12935-020-01363-z.
Additional file 1: Table S1. Correlation between PVT1 expression and clinicopathological parameters of patients.

Additional file 2: Figure S1. The predicted target miRNAs of PVT1 and their expression in PCa tissues and si-PVT1-transfected PCa cells. (A) The potential binding site between PVT1 and target miRNAs were analyzed by Starbase. (B) The expression of target miRNAs, including miR-515-5p, miR24-3p, miR-512-3p, miR-15a-5p, miR-21-5p and miR-17-5p, was detected using qRT-PCR in PCa tissues and normal tissues. (C) The expression of miR-515-5p, miR-24-3p, miR-512-3p and miR-15a-5p in $22 R V 1$ and DU145 cells transfected with si-PVT1 or si-NC was detected by qRT-PCR. ${ }^{*} P<0.05$.

Additional file 3: Figure S2. KIF23 overexpression rescued the effects of PVT1 knockdown. 22RV1 and DU145 cells were transfected with si-PVT1 or si-VT1 + oe-KIF23, with si-NC or or si-PVT1 + vector as the control. (A andB) The expression of KIF23 in these transfected cells was detected by qRT-PCR an western blot. (C) Cell proliferation was assessed by MTT assay. (D) Cell apoptosis was monitored by flow cytometry assay. (E and F) Cell migration and cell invasion in these transfected cells were investigated by transwell assay. (G) The expression of Vimentin, E-cad, Caspase-3 and CyclinD1 was quantified by western blot in these transfected cells. ${ }^{*} P<0.05$, ${ }^{*} P<0.01$.

Additional file 4: Figure S3. The expression of PVT1 in DU145 cells transfected with sh-PVT1 was notably declined. (A) The expression of PVT1 in DU145 cells transfected with sh-PVT1 or sh-NC was measured using qRT-PCR. ${ }^{*} P<0.05$. 


\section{Abbreviations}

PCa: Prostate cancer; IncRNAs: Long non-coding RNAs; miR-15a-5p: MicroRNA15a-5p; KIF23: Kinesin family member 23; qRT-PCR: Quantitative real-time polymerase chain reaction; EMT: Epithelial-mesenchymal transition; RPMI: Roswell Park Memorial Institute 1640; FBS: Fetal bovine serum; DMEM: Dulbecco's Modified Eagle Medium.

\section{Acknowledgements}

Not applicable.

\section{Authors' contributions}

All authors made substantial contribution to conception and design, acquisition of the data, or analysis and interpretation of the data; take part in drafting the article or revising it critically for important intellectual content; gave final approval of the revision to be published; and agree to be accountable for all aspect of the work. All authors read and approved the final manuscript.

\section{Funding}

No funding was received.

\section{Availability of data and materials}

The analyzed data sets generated during the present study are available from the corresponding author on reasonable request.

\section{Ethics approval and consent to participate}

The present study was approved by the ethical review committee of the Huaihe Hospital of Henan University.

\section{Patient consent for publication}

Not applicable.

\section{Competing interests}

The authors declare that they have no competing interests.

\section{Author details}

${ }^{1}$ Department of Telemedicine and Internet Medical Center, The Huaihe Hospital of Henan University, No. 115 Ximen Avenue, Kaifeng 475000, Henan, China.

${ }^{2}$ Department of Urology Surgery, The Huaihe Hospital of Henan University, Kaifeng, Henan, China.

\section{Received: 11 March 2020 Accepted: 17 June 2020}

Published online: 02 July 2020

\section{References}

1. Attard G, Parker C, Eeles RA, Schroder F, Tomlins SA, Tannock I, Drake CG, de Bono JS. Prostate cancer. Lancet. 2016;387(10013):70-82.

2. Marugame T, Katanoda K. International comparisons of cumulative risk of breast and prostate cancer, from cancer incidence in five continents, Vol. VIII. Jpn J Clin Oncol. 2006;36(6):399-400.

3. Torre LA, Bray F, Siegel RL, Ferlay J, Lortet-Tieulent J, Jemal A. Global cancer statistics, 2012. CA Cancer J Clin. 2015:65(2):87-108.

4. Lee J, Demissie K, Lu SE, Rhoads GG. Cancer incidence among KoreanAmerican immigrants in the United States and native Koreans in South Korea. Cancer Control. 2007;14(1):78-85.

5. Zhu Y, Yang XQ, Han CT, Dai B, Zhang HL, Shi GH, Wang CF, Ye DW. Pathological features of localized prostate cancer in China: a contemporary analysis of radical prostatectomy specimens. PLOS ONE. 2015;10(3):e0121076.

6. Yuan X, Cai C, Chen S, Chen S, Yu Z, Balk SP. Androgen receptor functions in castration-resistant prostate cancer and mechanisms of resistance to new agents targeting the androgen axis. Oncogene. 2014:33(22):2815-25.

7. Mercer TR, Dinger ME, Mattick JS. Long non-coding RNAs: insights into functions. Nat Rev Genet. 2009;10(3):155-9.

8. Wilusz JE, Sunwoo H, Spector DL. Long noncoding RNAs: functional surprises from the RNA world. Genes Dev. 2009;23(13):1494-504.

9. Tano K, Akimitsu N. Long non-coding RNAs in cancer progression. Front Genet. 2012:3:219.
10. Wu X, Xiao Y, Zhou Y, Zhou Z, Yan W. IncRNA SNHG20 promotes prostate cancer migration and invasion via targeting the miR-6516-5p/ SCGB2A1 axis. Am J Transl Res. 2019:11(8):5162-9.

11. Ren S, Liu Y, Xu W, Sun Y, Lu J, Wang F, Wei M, Shen J, Hou J, Gao X, et al. Long noncoding RNA MALAT-1 is a new potential therapeutic target for castration resistant prostate cancer. J Urol. 2013;190(6):2278-87.

12. Jin K, Wang S, Zhang Y, Xia M, Mo Y, Li X, Li G, Zeng Z, Xiong W, He Y. Long non-coding RNA PVT1 interacts with MYC and its downstream molecules to synergistically promote tumorigenesis. Cell Mol Life Sci. 2019;76(21):4275-89.

13. Lu D, Luo P, Wang Q, Ye Y, Wang B. IncRNA PVT1 in cancer: a review and meta-analysis. Clinica chimica acta Int J Clin Chem. 2017;474:1-7.

14. Ghafouri-Fard S, Omrani MD, Taheri M. Long noncoding RNA PVT1: a highly dysregulated gene in malignancy. J Cell Physiol. 2019;76(21):4275-89.

15. Sun BK, Tsao H. Small RNAs in development and disease. J Am Acad Dermatol. 2008;59(5):725-37 (quiz 38-40)

16. Hou X, Wen J, Ren Z, Zhang G. Non-coding RNAs: new biomarkers and therapeutic targets for esophageal cancer. Oncotarget. 2017:8(26):43571-8.

17. Yuan Y, Jiaoming L, Xiang W, Yanhui L, Shu J, Maling G, Qing M. Analyzing the interactions of mRNAs, miRNAs, IncRNAs and circRNAs to predict competing endogenous RNA networks in glioblastoma. J Neurooncol. 2018;137(3):493-502.

18. Mavrogiannis AV, Kokkinopoulou I, Kontos CK, Sideris DC. Effect of vinca alkaloids on the expression levels of microRNAs targeting apoptosis-related genes in breast cancer cell lines. Curr Pharm Biotechnol. 2018;19(13):1076-86

19. Jiang L, Wu Z, Meng X, Chu X, Huang H, Xu C. LncRNA HOXA-AS2 facilitates tumorigenesis and progression of papillary thyroid cancer by modulating the miR-15a-5p/HOXA3 axis. Hum Gene Ther. 2019;30(5):618-31.

20. Wang ZM, Wan XH, Sang GY, Zhao JD, Zhu QY, Wang DM. miR-15a-5p suppresses endometrial cancer cell growth via Wnt/beta-catenin signaling pathway by inhibiting WNT3A. Eur Rev Med Pharmacol Sci. 2017;21(21):4810-8.

21. Takahashi S, Fusaki N, Ohta S, I wahori Y, lizuka Y, Inagawa K, Kawakami Y, Yoshida K, Toda M. Downregulation of KIF23 suppresses glioma proliferation. J Neurooncol. 2012;106(3):519-29.

22. Kato T, Lee D, Wu L, Patel P, Young AJ, Wada H, Hu HP, Ujiie H, Kaji M, Kano S, et al. Kinesin family members KIF11 and KIF23 as potential therapeutic targets in malignant pleural mesothelioma. Int J Oncol. 2016;49(2):448-56.

23. Kato T, Wada H, Patel P, Hu HP, Lee D, Ujiie H, Hirohashi K, Nakajima T, Sato M, Kaji M, et al. Overexpression of KIF23 predicts clinical outcome in primary lung cancer patients. Lung Cancer. 2016;92:53-61.

24. Li XL, Ji YM, Song R, Li XN, Guo LS. KIF23 promotes gastric cancer by stimulating cell proliferation. Dis Markers. 2019;2019:9751923.

25. Wan L, Tian Y, Zhang R, Peng Z, Sun J, Zhang W. MicroRNA-103 confers the resistance to long-treatment of adriamycin to human leukemia cells by regulation of COP1. J Cell Biochem. 2018;119(5):3843-52.

26. Siegel RL, Miller KD, Jemal A. Cancer statistics, 2018. CA Cancer J Clin. 2018:68(1):7-30.

27. Xu YH, Deng JL, Wang G, Zhu YS. Long non-coding RNAs in prostate cancer: functional roles and clinical implications. Cancer Lett. 2019;464:37-55.

28. Wan B, Wu HY, Lv DJ, Zhou XM, Zhong LR, Lei B, Zhang SB, Mao XM. Downregulation of IncRNA PVT1 expression inhibits proliferation and migration by regulating p38 expression in prostate cancer. Oncol Lett. 2018:16(4):5160-6.

29. Chang Z, Cui J, Song Y. Long noncoding RNA PVT1 promotes EMT via mediating microRNA-186 targeting of Twist1 in prostate cancer. Gene. 2018;654:36-42.

30. Liu HT, Fang L, Cheng YX, Sun Q. LncRNA PVT1 regulates prostate cancer cell growth by inducing the methylation of miR-146a. Cancer Med. 2016:5(12):3512-9.

31. Jin W, Chen F, Wang K, Song Y, Fei X, Wu B. miR-15a/miR-16 cluster inhibits invasion of prostate cancer cells by suppressing TGF-beta signaling pathway. Biomed Pharmacother. 2018;104:637-44. 
32. Zidan HE, Abdul-Maksoud RS, Elsayed WSH, Desoky EAM. Diagnostic and prognostic value of serum miR-15a and miR-16-1 expression among Egyptian patients with prostate cancer. IUBMB Life. 2018;70(5):437-44.

33. Long J, Jiang C, Liu B, Fang S, Kuang M. MicroRNA-15a-5p suppresses cancer proliferation and division in human hepatocellular carcinoma by targeting BDNF. Tumour Biol. 2016;37(5):5821-8.

34. Gao D, Zhang Y, Zhu M, Liu S, Wang X. miRNA expression profiles of HPVinfected patients with cervical cancer in the uyghur population in china. PLoS ONE. 2016;11(10):e0164701.

35. Kontos CK, Tsiakanikas P, Avgeris M, Papadopoulos IN, Scorilas A. miR15a-5p, a novel prognostic biomarker, predicting recurrent colorectal adenocarcinoma. Mol Diagn Ther. 2017;21(4):453-64.

36. Sun $X$, Jin Z, Song $X$, Wang J, Li Y, Qian X, Zhang Y, Yin Y. Evaluation of KIF23 variant 1 expression and relevance as a novel prognostic factor in patients with hepatocellular carcinoma. BMC Cancer. 2015;15:961.
37. Gao H, Chen X, Cai Q, Shang Z, Niu Y. Increased KIF4A expression is a potential prognostic factor in prostate cancer. Oncol Lett. 2018;15(5):7941-7.

38. Sekino Y, Oue N, Shigematsu Y, Ishikawa A, Sakamoto N, Sentani K, Teishima J, Matsubara A, Yasui W. KIFC1 induces resistance to docetaxel and is associated with survival of patients with prostate cancer. Urol Oncol. 2017;35(1):31.e13-31, e20.

39. Piao XM, Byun YJ, Jeong P, Ha YS, Yoo ES, Yun SJ, Kim WJ. Kinesin family member $11 \mathrm{mRNA}$ expression predicts prostate cancer aggressiveness. Clin Genitourin Cancer. 2017;15(4):450-4.

\section{Publisher's Note}

Springer Nature remains neutral with regard to jurisdictional claims in published maps and institutional affiliations.
Ready to submit your research? Choose BMC and benefit from:

- fast, convenient online submission

- thorough peer review by experienced researchers in your field

- rapid publication on acceptance

- support for research data, including large and complex data types

- gold Open Access which fosters wider collaboration and increased citations

- maximum visibility for your research: over $100 \mathrm{M}$ website views per year

At BMC, research is always in progress.

Learn more biomedcentral.com/submissions 